\title{
Comparison of Laser Performance for Diode-Pumped Tm:YLF of Various Doping Concentrations
}

\author{
J. I. Mackenzie, S. So, D. P. Shepherd, and W. A. Clarkson \\ Optoelectronics Research Centre, University of Southampton, Southampton SO17 1BJ, UK. \\ jim@orc.soton.ac.uk
}

\begin{abstract}
Single-end-pumped laser performance of 2, 4, and 6at.\% Tm-doped YLF rods is reported. For the pumping configuration employed, crystal fracture was observed to occur for a thermal load per unit absorption length of $\sim 13 \mathrm{~W} / \mathrm{cm}$. The 2 at. $\%$ Tm-doped crystal was found to have a quantum yield of $\sim 90 \%$ of that of $4 \mathrm{at} . \% \mathrm{Tm}$. However, due to a lower thermal loading density, the maximum possible incident pump power is predicted to be $>60 \%$ higher, hence offering a greater output power per rod for the lower doping concentration. Power scaling considerations are discussed with reference to crossrelaxation, upconversion, and thermal loading of the host crystal.

OCIS codes: (140.3580) Lasers, solid-state; (140.3070) Infrared and far-infrared lasers; (140.3480) Lasers, diode-pumped
\end{abstract}

\section{Introduction}

Two-micron $\mathrm{Tm}^{3+}$ lasers are of interest for many applications in the scientific, defence, and medical fields. Thulium readily substitutes into many crystal hosts that are suitable for high-average-power laser systems and it has an absorption band at $\sim 0.8 \mu \mathrm{m}$ allowing excitation with commercially available highpower laser diodes [1]. Thulium-doped YLF has been investigated by several groups, with several tens of Watts being reported by Budni et. al. [2], and Dergachev et. al. [3], in end- and side-pumped configurations respectively. YLF, although recognised as a good laser host for its spectroscopic properties, is a relatively fragile crystal with a low stress-fracture limit. Thermally-induced fracture has been reported to occur when end-pumping Tm:YLF with $792 \mathrm{~nm}$ diodes and beam-waist pump intensities of $>5 \mathrm{kWcm}^{-2}$ [2]. This limit can be increased by $\sim 60 \%$ via the use of diffusion bonded undoped end caps [4]. Alternatively, for power scaling, reducing the doping concentration of the host crystal and thus the absorption coefficient, albeit with higher demands on the pump-source brightness, leads to smaller temperature excursions and thus reduces stress on the rod-air interface. However, in the case of Tm-doped solid-state lasers there are conflicting requirements since at high doping levels Tm lasers benefit from an increase in the quantum efficiency due to the 'two-for-one' cross-relaxation (selfquenching) process, ${ }^{3} \mathrm{~F}_{4},{ }^{3} \mathrm{H}_{6} \rightarrow{ }^{3} \mathrm{H}_{4},{ }^{3} \mathrm{H}_{4}$, which becomes less efficient as the $\mathrm{Tm}$ concentration is reduced leading to a reduction in the slope efficiency and increased thermal loading.

Another important factor to consider is the three-level-nature of Tm:YLF when operating in the $2 \mu \mathrm{m}$ regime, which requires that $\sim 10 \%$ of the lower manifold population is excited into the upper laser level to obtain transparency at the lasing wavelength. One consequence of large excited-state population densities is the exacerbation of energy-transfer-upconversion (ETU), which reduces the upper laser level lifetime and thus increases laser threshold. This process is also dependent on the density of the excited $\mathrm{Tm}$ ions and therefore the concentration and pump intensity. Furthermore, ETU contributes to the amount of heat generated in the crystal, thereby impacting on the stress in the laser crystal, the lower laser level population density, and consequently the required population inversion. The spectroscopic ETU rate parameters can be determined directly as a function of the $\mathrm{Tm}^{3+}$ doping concentration from a measurement of the fluorescence decay from the upper level. However more useful information for the laser designer can be obtained directly from a simple comparative study of the performance of Tm:YLF 
lasers with different Tm concentrations, since this encapsulates all the inter-dependent processes. Ultimately therefore, when choosing a Thulium concentration there is a trade off between the beneficial cross-relaxation, detrimental ETU, and thermal loading density within the laser crystal.

To allow a strategy to be formulated for scaling of Tm:YLF laser powers requires knowledge of several key design parameters, of which there are few to be found in the literature. Reported here are preliminary findings of a comparative study of laser performance for singly-end-pumped Tm:YLF rods with a range of doping concentrations.

\section{Experimental set-up}

The $3 \mathrm{~mm}$ diameter (a-cut) Tm:YLF laser rods ${ }^{\mathrm{i}}$ investigated in our study were doped with 2, 4, and 6 at. $\% \mathrm{Tm}^{3+}$. Both end faces of the rods were antireflection coated for pump and lasing wavelengths at $792 \mathrm{~nm}$ and $\sim 1.9 \mu \mathrm{m}$ respectively, and the rod lengths were selected to be two absorption lengths at the pump wavelength (i.e. $24 \mathrm{~mm}, 12 \mathrm{~mm}$, and $8 \mathrm{~mm}$ respectively). All rods were mounted in water-cooled copper blocks to allow effective heat removal whilst pumped by high-power diode-laser arrays.

For this study a simple two-mirror resonator configuration was employed, comprising a plane pump in-coupling mirror with high reflectivity at the lasing wavelength and high transmission at the pump wavelength, and a concave output coupler with a $200 \mathrm{~mm}$ radius of curvature. A cavity length of $100-120 \mathrm{~mm}$ was selected to produce a calculated average $\mathrm{TEM}_{\mathrm{oo}}$ beam radius in the YLF crystal (under unpumped conditions) of $\sim 260 \mu \mathrm{m}$, which was expected to increase by up to $50 \%$ at the maximum available pump power due to the effect of the weak negative thermal lensing in Tm:YLF. The pump beam was focussed to a waist radius, $\mathrm{w}_{\mathrm{p}} \approx 460 \mu \mathrm{m}$, roughly equal to that required for confocal pumping of the 2 at. $\%$ rod (i.e. $\left.\mathrm{w}_{\mathrm{p}}=\left(\mathrm{M}^{2} \lambda \mathrm{L} / 2 \mathrm{n} \pi\right)^{1 / 2}\right)$ and positioned approximately at the mid-point of each laser rod respectively. In this way all of the Tm:YLF lasers were deliberately operated in a multi-transverse mode regime and under the same conditions, as such the effects of thermally-induced lens aberration on output power and efficiency were minimised, and a relative comparison between the performance for each doping concentration could be made.

Two diode-laser pump sources were employed in this study (Fig. 1): the first consisted of two low-fill-factor water-cooled diode-bars and the second a 6-bar low-fill-factor diode stack. Each bar, for
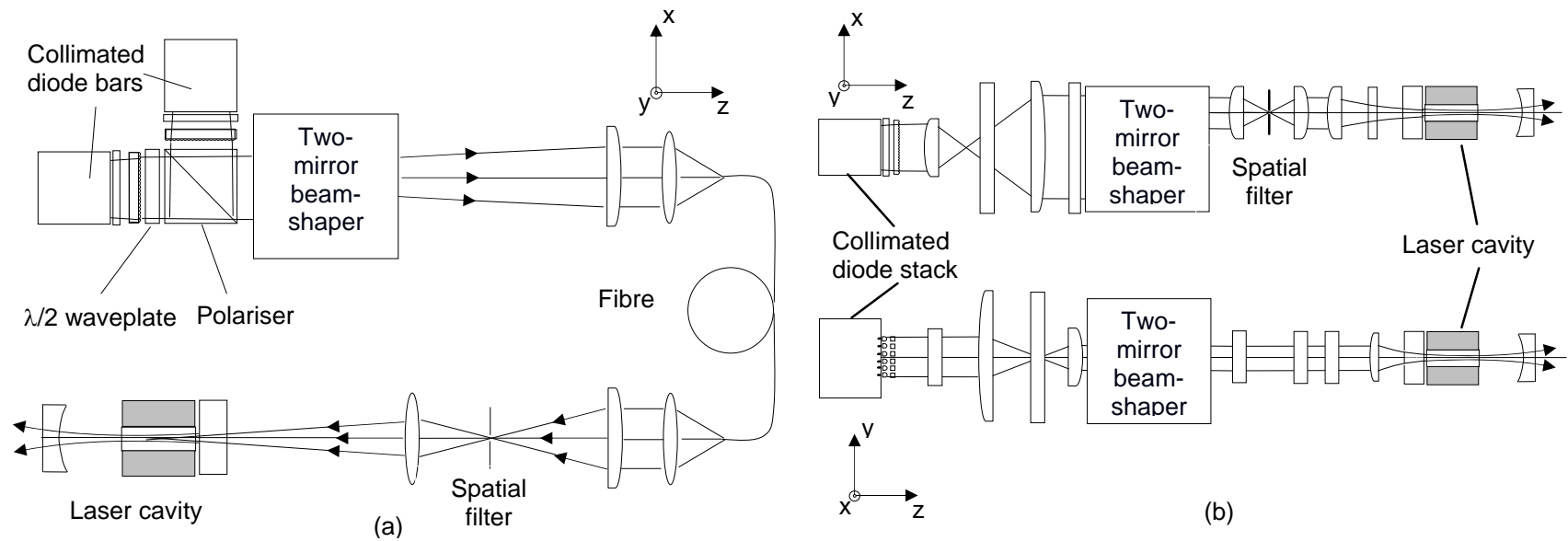

Fig. 1: Experimental configuration with (a) diode-bar pumping and (b) diode-stack pumping.

\footnotetext{
i Impex High-Tech, Rheine, Germany
} 
both of the sources, was collimated in the fast and slow-axes with micro-lenses. The single diode bars were then polarisation combined to produce a single beam. Two-mirror beam-shapers [5] were employed to re-format the beams with roughly equal beam-quality factors in the orthogonal planes. For the polarisation-coupled bars, the resulting beam-quality was $\mathrm{M}_{\mathrm{x}}{ }^{2} \approx 80$ (parallel to the array) and $\mathrm{M}_{\mathrm{y}}{ }^{2} \approx 52$ (perpendicular to the array) with a maximum power of $\sim 52 \mathrm{~W}$. In contrast the final beam-shaped diode stack beam-quality was $\mathrm{M}_{\mathrm{x}}{ }^{2} \approx 160$ and $\mathrm{M}_{\mathrm{y}}{ }^{2} \approx 100$ with a maximum power of $\sim 265 \mathrm{~W}$. A uniform pump distribution was desirable for these comparative studies, hence the output beam from the diode-bar pump source was 'homogenised' by coupling into a multi-mode fibre with a core diameter of $365 \mu \mathrm{m}$ and 0.22 NA. A spatial filter was used to reduce the $\mathrm{M}^{2}$ value to $\sim 100$ allowing relatively tight pump focussing with minimal diffraction spreading even in the longest Tm:YLF rod under investigation, limiting the maximum available pump power to $\sim 33 \mathrm{~W}$. To produce a similar beam-quality from the diode stacks, a spatial filtering slit was employed in the $\mathrm{x}$-axis only, limiting the available incident power to $\sim 115 \mathrm{~W}$, which was more than sufficient for our study.

\section{Results and Discussion}

Fig. 2(a) shows the output power versus incident pump power for all three Tm:YLF rods using a 13.5\% output coupler. Less than $10 \%$ output coupling resulted in a longer operating wavelength away from the peak of the $\sigma$-polarised emission cross section at $1.91 \mu \mathrm{m}$. A linear fit to the data points for pump powers greater than three times threshold has been used to calculate the slope efficiency thus providing a good basis for comparison when operating in the saturated regime. Similar performance curves are shown in Fig. 2(b) for diode-stack pumping, apart from the 6at.\% crystal which fractured whilst diode-bar pumping at an incident pump power of $20.5 \mathrm{~W}$. Furthermore the fracture limit for the 4 at. $\%$ crystal was found when diode-stack pumping at an incident power of $45.8 \mathrm{~W}$. The pump absorption efficiencies at threshold were $\sim 82 \%$ and $\sim 84 \%$ respectively for Fig. 2 (a) and (b), this small difference can be attributed to the linearly-polarised output of the diode stack source in contrast to the "un-polarised" output from the fibre-coupled diode-bars.

The laser output was determined to be $\sigma$-polarised with a wavelength of $1.91 \mu \mathrm{m}$. Thermal lensing is known to be weaker for $\sigma$-polarised light in YLF crystals due to a lower value for $\mathrm{dn} / \mathrm{dT}$ than for the orthogonal $\pi$-polarisation. The weak nature of thermal lensing in the Tm:YLF laser is apparent, since there is no evidence of a thermally-induced roll-over in the output power.

As illustrated by Fig. 2, there is clearly a very pronounced effect of the Tm doping level on performance. Given that all three Tm:YLF rods were operated under nearly identical conditions in terms of cavity loss, pump beam and laser mode sizes and re-absoprtion loss, we can draw the following conclusions: (a) The difference in slope efficiency for the $2 \mathrm{at} \%$ and $4 \mathrm{at} \% \mathrm{Tm}$-doped rods is primarily due to a difference in the cross-relaxation efficiency. We can therefore conclude that the pumping quantum efficiency is approximately $\sim 10 \%$ higher for the 4 at $\%$ doping level than for the $2 \mathrm{at} . \%$ doping level. (b) The difference in threshold pump power for the three rods is due to the combined effects of ETU and cross-relaxation. It is observed that the $2 \mathrm{at} . \%$ and $4 \mathrm{at} . \% \mathrm{Tm}^{3+}$ doped rods have comparable thresholds indicating that the higher cross-relaxation efficiency for the $4 \mathrm{at} . \% \mathrm{Tm}^{3+}$ doped rod is offset by a higher loss due to ETU compared to the 2at.\% $\mathrm{Tm}^{3+}$ doped rod. On the other hand the 6at.\% $\mathrm{Tm}^{3+}$ doped rod had a $\sim 1.5$ times higher threshold indicating that ETU has a very significant impact on its performance. In the case of the two higher doping concentrations the maximum output powers were limited by stress fracture. This is expected to be the case for the 2 at.\% doped crystal as well and an estimation of the maximum pump power can be calculated from the comparative parameters reported here. 


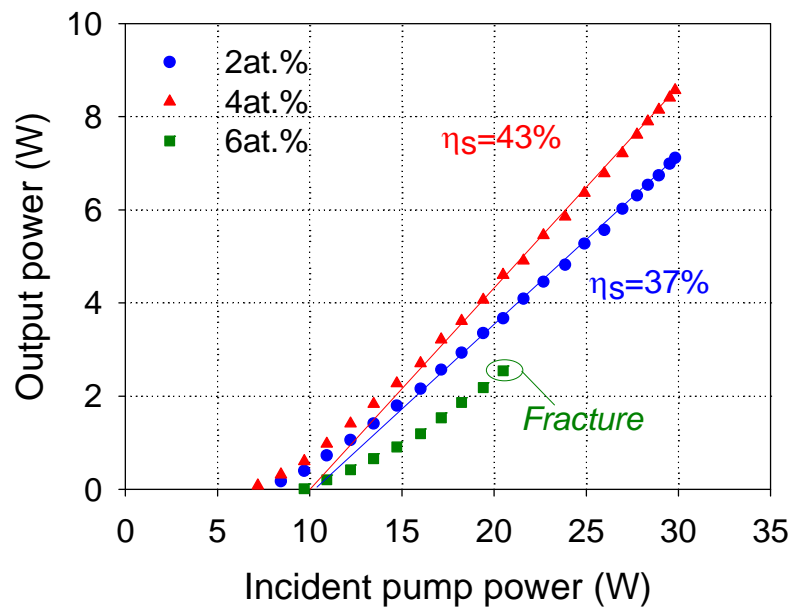

(a)

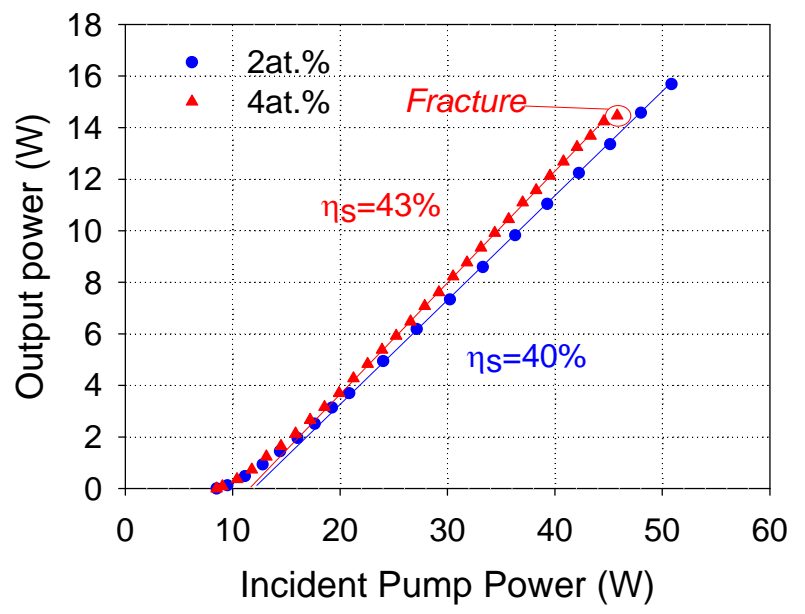

(b)

Fig. 2. Tm:YLF laser performance for various doping concentrations for (a) diode-bar and (b) diode-stack pumping.

An approximate value for the stress-fracture pump power limit can be obtained by comparing the thermal load per unit length for the three doping concentrations. The maximum stress, $\sigma_{\max }$, which YLF can tolerate in an end-pumped configuration before fracture occurs is dependent upon its material parameters, the pump beam size and the rod diameter [6]. The variable parameters for comparison here are the absorption length, $\alpha_{a b s}$ and the total maximum heat load, $P_{h_{-} \max }$ :

$$
\sigma_{\max } \propto \alpha_{a b s} P_{h_{-} \max }
$$

where $P_{h}$ is the power converted to heat when the pump power exceeds that required for threshold, and is given by:

$$
P_{h}=\left(1-\eta_{Q Y} \eta_{S t}\right)\left(P_{a b s}-P_{E T U}\right)+P_{E T U}
$$

where $\eta_{Q Y}$ is the quantum yield, $\eta_{S t}$ the Stokes efficiency, $P_{E T U}$ the amount of absorbed power channelled into ETU, which is clamped at threshold and is totally transferred to the crystal lattice as heat, and $P_{a b s}$ the absorbed pump power. At this point an estimation of the quantum yield as a function of doping concentration is required, which has been calculated (Fig. 3) following the method of Honea et. al. [7], where the reported Tm:YLF lifetime measurements by Armagan et. al. [8] for various doping concentrations and the ${ }^{3} \mathrm{~F}_{4} \rightarrow{ }^{3} \mathrm{H}_{4}$ branching ratio after Walsh et. al. [9] have been used. The ratio of the predicted quantum yield values for 2 and 4at.\% Tm (see Fig. 3) and the measured slope efficiencies are in good agreement. Therefore we can be confident in utilising the predicted quantum yield values to estimate the maximum heat load for the various doping concentrations investigated. The method employed is as follows, first one has to evaluate the threshold pump power that would be expected in the absence of ETU. This is achieved by simply scaling the threshold pump power measured for the 2at.\% crystal by the ratio of 1.8 , the quantum yield for 2 at. $\%$, with the quantum yield for the doping concentration of interest. The difference between the measured threshold power and the predicted threshold pump power without ETU represents $P_{E T U}$. Note it is necessary to assume $P_{E T U}$ is negligible for 2 at. $\%$ Tm with respect to the threshold pump power, such an assumption is warranted noting the small difference in threshold between 2 and 4 at.\% Tm in contrast to that for 6 at. $\% \mathrm{Tm}$.

Equation (2) and the parameters listed in Table 1, give the heating power per unit absorption length as presented in Fig. 4. The predicted maximum incident pump power, for the configuration detailed here, at which crystal fracture is predicted to occur for each doping concentration is indicated in the final column of Table 1. It has been assumed; (a) the threshold absorption efficiency was that for the 
diode-bar pumping experiments, (b) there was negligible ETU for 2at.\% Tm, thus enabling the estimation of the threshold power without ETU for each doping level, and (c) the calculated thermal load at which the 6 at. $\%$ crystal failed, $\sim 13 \mathrm{~W} / \mathrm{cm}$, is a benchmark for the other crystals.

Table 1. Comparison summary of the parameters used to calculate the thermal loading in the diode-bar pumped Tm:YLF

\begin{tabular}{ccccccc}
\hline $\begin{array}{c}\text { Doping conc. } \\
\text { (At.\%) }\end{array}$ & $\mathrm{P}_{\text {th }}$ meas. $(\mathrm{W})$ & $\eta_{\text {QY }}$ predicted & $\begin{array}{c}\mathrm{P}_{\text {th }} \text { estim. } \\
\text { without ETU }\end{array}$ & $\begin{array}{c}\mathrm{P}_{\text {ETU }} * \\
(\mathrm{~W})\end{array}$ & $\begin{array}{c}\alpha_{\text {abs }} \mathrm{P}_{\mathrm{h}} @ 6 \mathrm{P}_{\text {frac }} \text { for } \\
6 \mathrm{At} . \%(\mathrm{~W} / \mathrm{cm})\end{array}$ & $\begin{array}{c}\text { Predicted } \mathrm{P}_{\text {inc }} \text { limit } \\
(\mathrm{W})\end{array}$ \\
\hline 2 & 7.2 & 1.8 & 7.2 & $\sim 0$ & 3.6 & 74.6 \\
4 & 6.8 & 1.95 & 6.6 & 0.13 & 5.6 & 48.5 \\
6 & 9.7 & 1.97 & 6.6 & 2.6 & 13.0 & 20.5 (meas.) \\
\hline
\end{tabular}

* Note the absorption efficiency of $82 \%$ has been included here.

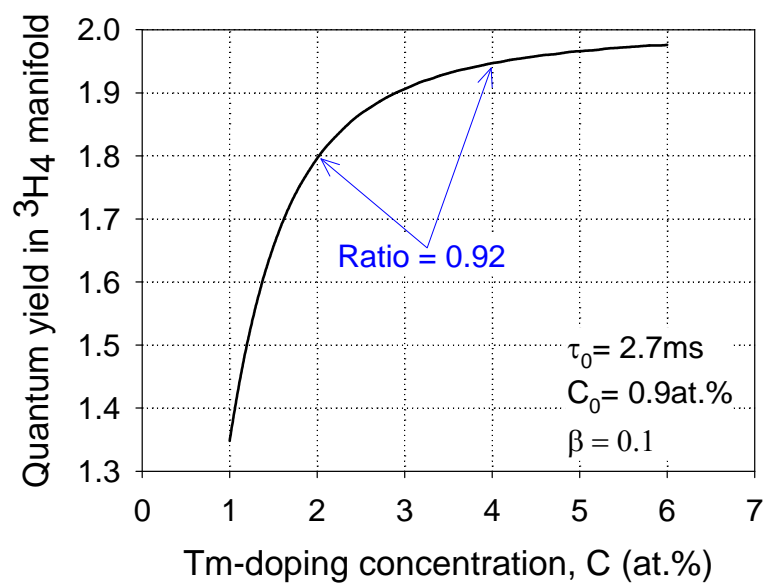

Fig. 3. Estimated quantum yield versus Tm concentration. $\tau_{0}$ is the un-quenched lifetime of the ${ }^{3} \mathrm{~F}_{4}$ manifold, $\mathrm{C}_{0}$ a characteristic doping concentration, and $\beta$ the branching ratio for ${ }^{3} \mathrm{~F}_{4} \rightarrow{ }^{3} \mathrm{H}_{4}$ [7-9].

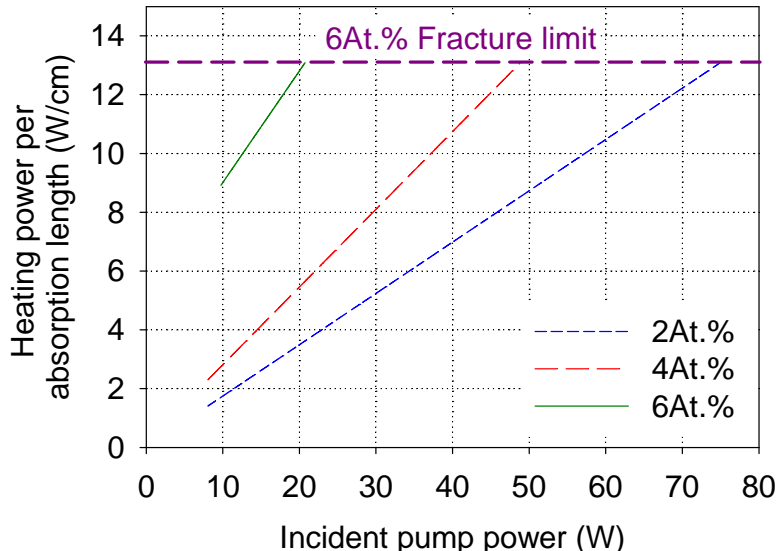

Fig. 4.Predicted heating power versus incident pump power for the various doping concentrations

Considering Fig. 4. it is important to note that equation (2) is valid only above threshold, i.e. when $P_{E T U}$ is clamped, below threshold both the quantum defect heating and heating due to upconversion vary with pump power and would tend to zero for no incident pump power. Comparing the measured incident pump power at which fracture was observed for 4at.\% Tm, i.e. 45.8W (Fig.2(b)), with the predicted value, $48.5 \mathrm{~W}$ (Fig. 4.), we see that there is good agreement. Further support of this method is found when accounting for the higher threshold condition measured for diode-stack pumping, Fig 2(b). The higher threshold was attributed to the difference in the absorption efficiency and pump distribution within the Tm:YLF crystals for the two pump sources. As such a thermal load per absorption length of $13 \mathrm{~W} / \mathrm{cm}$, corresponding to the 6 at.\% fracture limit, is calculated for the 4at.\% crystal, which corresponds to an incident power of $45 \mathrm{~W}$ and is in excellent agreement with the measured value of fracture-limit of $45.8 \mathrm{~W}$ for diode-stack pumping. This provides a good level of confidence for the predicted maximum pump power limit for the $2 \mathrm{at} . \%$ Tm:YLF crystal of $75 \mathrm{~W}$, which could generate $\sim 25 \mathrm{~W}$ of output power from a single standard single-end-pumped rod.

\section{Conclusions}

A comparison of laser performance for various doping concentrations of Tm:YLF has been reported. For the end-pumped configuration described, a thermal load per absorption length of $\sim 13 \mathrm{~W} / \mathrm{cm}$ represents 
the stress-fracture limit for Tm:YLF rods. Consequently at the few tens of Watts pump power levels, the 4at.\% Tm:YLF rod offered the best performance in terms of maximum output power and overall efficiency. However, despite having a $\sim 10 \%$ lower quantum yield, the 2 at. $\%$ Tm:YLF crystal, by virtue of a lower thermal loading density and therefore a much higher fracture-limit at the pump wavelength of $\sim 792 \mathrm{~nm}$, appears to offer better prospects for end-pumped power scaling.

\section{Acknowledgements}

The authors would like to acknowledge Dr G. Rustad for very helpful discussions regarding earlier work on this topic. This work was funded by QinetiQ and carried out under the auspices of the UK Ministry of Defence Corporate Research Programme.

\section{References}

[1] S. A. Payne, L. L. Chase, L. K. Smith, W. L. Kway, and W. F. Krupke, "Infrared Cross-Section Measurements for Crystals Doped with $\mathrm{Er}^{3+}, \mathrm{Tm}^{3+}$, and $\mathrm{Ho}^{3+}, "$ IEEE Journal of Quantum Electronics, vol. 28, pp. 2619-2630, 1992.

[2] P. A. Budni, M. L. Lemons, J. R. Mosto, and E. P. Chicklis, "High-power/high-brightness diode-pumped $1.9 \mu \mathrm{m}$ Thulium and resonantly pumped $2.1 \mu \mathrm{m}$ Holmium lasers," IEEE Journal of Selected Topics in Quantum Electronics, vol. 6, pp. 629-635, 2000.

[3] A. Dergachev, K. Wall, and P. F. Moulton, "A CW side-pumped Tm : YLF laser," in Advanced SolidState Lasers, Proceedings, vol. 68, OSA Trends in Optics and Photonics, 2002, pp. 343-346.

[4] M. Tsunekane, N. Taguchi, T. Kasamatsu, and H. Inaba, "Analytical and experimental studies on the characteristics of composite solid-state laser rods in diode-end-pumped geometry," IEEE Journal of Selected Topics in Quantum Electronics, vol. 3, pp. 9-18, 1997.

[5] W. A. Clarkson and D. C. Hanna, "Two-mirror beam-shaping technique for high-power diode bars," Optics Letters, vol. 21, pp. 375-377, 1996.

[6] L. Yan and C. H. Lee, "Thermal effects in end-pumped Nd:phosphate glasses," Journal of Applied Physics, vol. 75, pp. 1286-1292, 1994.

[7] E. C. Honea, R. J. Beach, S. B. Sutton, J. A. Speth, S. C. Mitchell, J. A. Skidmore, M. A. Emanuel, and S. A. Payne, "115-W Tm:YAG diode-pumped solid-state laser," IEEE Journal of Quantum Electronics, vol. 33, pp. 1592-1600, 1997.

[8] G. Armagan, A. M. Buoncristiani, A. T. Inge, and B. D. Bartolo, "Comparison of the spectroscopic properties of Tm and Ho in YAG and YLF crystals," in Advanced Solid-State Lasers, Proceedings, vol. 10, OSA Trends in Optics and Photonics, 1991, pp. 222-226.

[9] B. M. Walsh, N. P. Barnes, and B. Di Bartolo, "Branching ratios, cross sections, and radiative lifetimes of rare earth ions in solids: Application to $\mathrm{Tm}^{3+}$ and $\mathrm{Ho}^{3+}$ ions in LiYF4," Journal of Applied Physics, vol. 83, pp. 2772-2787, 1998. 